\title{
LETTER
}

\section{Impairment of hepatocellular excretory function, sepsis and liver insufficiency after liver resection}

\author{
Carlo Chiarla, Ivo Giovannini", Francesco Ardito, Maria Vellone, Gennaro Nuzzo and Felice Giuliante \\ See related research by Gonnert et al., http://ccforum.com/content/17/2/R67
}

We appreciated the article by Gonnert and colleagues on hyperbilirubinemia from septic hepatocellular excretory dysfunction [1], a still improperly characterized issue in clinical settings. We address postoperative liver resection patients with prominently conjugated ( $\sim 50$ to $70 \%$ conjugated) hyperbilirubinemia. Less relevant causes include transient insufficiency of remnant liver and prolonged intraoperative ischemia [2]; a threatening cause is occult sepsis.

The pattern may be similar: prominently conjugated hyperbilirubinemia, with normal/moderately altered aspartate aminotransferase and alanine aminotransferase, alkaline phosphatase and gamma-glutamyltransferase. In occult sepsis this involves the risk of dismissing hyperbilirubinemia as transient liver insufficiency expected to spontaneously recover, with dreadful consequences, also because sepsis and liver insufficiency might coexist [3]. In post-hepatectomy patients, interpretation of hyperbilirubinemia is challenged by unreliability of landmarks based on hepatobiliary enzymes. Aspartate aminotransferase and alanine aminotransferase increase due to par- enchymal transection/ischemia, and then decrease at different rates [4]; alkaline phosphatase and gammaglutamyltransferase (enzymes of cholestasis) markedly decrease, and then slowly increase reflecting liver regeneration $[2,5]$. We even observed isolated hyperbilirubinemia ( $>15.0 \mathrm{mg} / \mathrm{dl}, 50 \%$ conjugated) with near-normal alkaline phosphatase and gamma-glutamyltransferase and good clinical condition in occult sepsis from posthepatectomy biliary stenosis and initial cholangitis. Although this was partly obstructive cholestasis (a less pertinent example), it better emphasizes the need to promptly exclude sepsis or other complications as causes of hyperbilirubinemia, without relying on common clinical/biochemical criteria.

The light shed by Gonnert and colleagues [1] on septic hepatocellular excretory dysfunction has major translational implications: as obvious as the issue may appear to updated investigators, greater awareness of this deceitful presentation of sepsis may still avoid dangerous delays in treatment.

\section{Authors' response \\ Falk A Gonnert, Michael Bauer and Andreas Kortgen}

We appreciate the comments from Chiarla and colleagues since they describe a typical but challenging dilemma of diagnosing sepsis-associated liver dysfunction [6]. It is important to note that liver dysfunction occurs frequently and early in sepsis, often without hepatocellular injury [7]. Sepsis induces a reprogramming of metabolic functions in parallel with a severity-dependent disruption of phase I and phase II biotransformation and canalicular transport [8], whereby activation of PI3kinase-dependent signaling could be a pathogenetic mechanism [7]. Since these changes influence prognosis

\footnotetext{
* Correspondence: ivo.giovannini@rm.unicatt.it

CNR-IASI Center for Pathophysiology of Shock, Department of Surgical

Science, Catholic University of the Sacred Heart, Largo Agostino Gemelli 8, I-00168 Rome, Italy
}

and significantly precede conventional markers [7,9], there is an urgent need for new diagnostic strategies.

Nowadays, the plasma disappearance rate of indocyanine green is the best evaluated test to assess hepatic function. This test reflects excretory dysfunction yet underestimates impaired canalicular transport [9]. A recent report suggested the LiMAx test, a new non-invasive diagnostic tool for determining liver function based on a breath test [10]. Both tests are superior to conventional laboratory markers in predicting patient morbidity and mortality. In addition to the presented biophotonic techniques in our study, another future perspective in early diagnosing liver dysfunction in the critically ill might be plasma bile acids. In septic patients, levels of both 
conjugated and unconjugated chenodeoxycholic and taurodeoxycholic acid were increased on the day of diagnosis, showing a stronger correlation with 28-day mortality than bilirubin levels [7].

While there is still a need for improving our spectrum of diagnostic tools, another true challenge will be the development of specific therapeutic strategies for critically ill patients with liver dysfunction.

\section{Competing interests}

MB is member of the medical advisory board of Pulsion Medical Systems SE. AK received a study grant from Pulsion Medical Systems SE. MB and FAG hold a patent for application of polymethine fluorescent dyes for monitoring organ dysfunction. The invention further relates to a kit for determining an organ function with a marker dye. The remaining authors declare that they have no competing interests.

Received: 4 October 2013 Accepted: 17 March 2014

Published: 25 Mar 2014

\section{References}

1. Gonnert FA, Recknagel P, Hilger I, Claus RA, Bauer M, Kortgen A: Hepatic excretory function in sepsis: implications from biophotonic analysis of transcellular xenobiotic transport in a rodent model. Crit Care 2013, 17: R67.

2. Chiarla C, Giovannini I, Giuliante F, Vellone M, Ardito F, Masi A, Nuzzo G: Plasma bilirubin correlations in non-obstructive cholestasis after partial hepatectomy. Clin Chem Lab Med 2008, 46:1598-1601.

3. Nuzzo G, Giovannini I, Giuliante F, Ardito F, Chiarla C: Sepsis after liver resection: predisposition, clinical relevance and synergism with liver dysfunction. In Recent Advances in Liver Surgery. Edited by Dionigi R. Austin, TX: Landes Bioscience; 2009:245-259.

4. Giovannini I, Chiarla C, Giuliante F, Vellone M, Ardito F, Sarno G, Nuzzo G: Analysis of the components of hypertransaminasemia after liver resection. Clin Chem Lab Med 2007, 45:357-360.

5. Osada S, Saji S: The clinical significance of monitoring alkaline phosphatase level to estimate postoperative liver failure after hepatectomy. Hepatogastroenterology 2004, 51:1434-1438.

6. Marshall JC: The liver in sepsis: shedding light on the cellular basis of hepatocyte dysfunction. Crit Care 2013, 17:153.

7. Recknagel P, Gonnert FA, Westermann M, Lambeck S, Lupp A, Rudiger A Dyson A, Carre JE, Kortgen A, Krafft C, Popp J, Sponholz C, Fuhrmann V, Hilger I, Claus RA, Riedemann NC, Wetzker R, Singer M, Trauner M, Bauer M: Liver dysfunction and phosphatidylinositol-3-kinase signalling in early sepsis: experimental studies in rodent models of peritonitis. PLOS Med 2012, 9:e1001338.

8. Bauer M, Press AT, Trauner M: The liver in sepsis: patterns of response and injury. Curr Opin Crit Care 2013, 19:123-127.

9. Kortgen A, Paxian M, Werth M, Recknagel P, Rauchfuss F, Lupp A, Krenn CG, Muller D, Claus RA, Reinhart K, Settmacher U, Bauer M: Prospective assessment of hepatic function and mechanisms of dysfunction in the critically ill. Shock 2009, 32:358-365.

10. Kaffarnik MF, Lock JF, Vetter H, Ahmadi N, Lojewski C, Malinowski M, Neuhaus P, Stockmann M: Early diagnosis of sepsis-related hepatic dysfunction and its prognostic impact on survival: a prospective study with the LiMAx test. Crit Care 2013, 17:R259.

$10.1186 /$ cc13792

Cite this article as: Chiarla et al:: Impairment of hepatocellular excretory function, sepsis and liver insufficiency after liver resection. Critical Care $2014,18: 419$ 